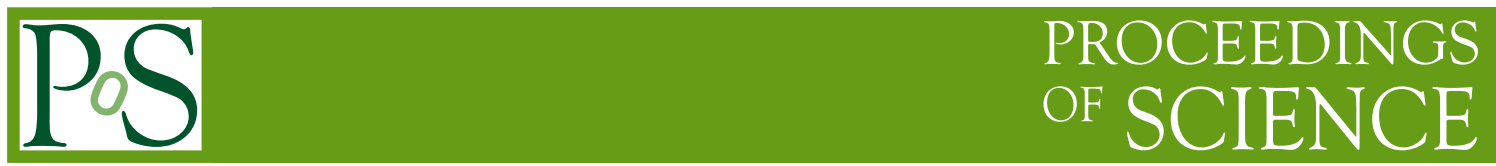

\title{
Universal chemical freeze-out as a phase transition signature
}

\author{
Ulrich Heinz ${ }^{* \dagger}$ and Gregory Kestin \\ Department of Physics, The Ohio State University, Columbus, OH 43210, USA \\ E-mail: heinz@mps.ohio-state.edu
}

\begin{abstract}
It is shown that kinetic freeze-out in relativistic heavy-ion collisions invariably entails a nontrivial dependence of the freeze-out temperature on the collision centrality. The centrality independence of the chemical freeze-out temperature observed in Au+Au collisions at RHIC is therefore inconsistent with the hypothesis that hadron abundances decouple kinetically from inelastic hadron-hadron interactions. On the other hand, it is consistent with the hypothesis that chemical decoupling is driven by the quark-hadron phase transition, and that the observed universal chemical freeze-out reflects its critical temperature, independent of the dynamical state of the collision fireball as it passes through the phase transition.
\end{abstract}

Critical Point and Onset of Deconfinement

July 3-6, 2006

Galileo Galilei Institute, Florence, Italy

\footnotetext{
* Speaker.

${ }^{\dagger}$ Work supported by the U.S. Department of Energy, grant DE-FG02-01ER41190.
} 


\section{Introduction}

From Lattice QCD [1] we know that strongly interacting matter of zero net baryon density undergoes a deconfining and chiral symmetry restoring phase transition from a hadron resonance gas at low temperatures to a quark-gluon plasma (QGP) at high temperature. The critical temperature of this transition has for several years been quoted [1] as $T_{c}=173 \pm 15 \mathrm{MeV}$ (with an associated critical energy density $e_{c} \approx 0.7 \mathrm{GeV} / \mathrm{fm}^{3}$ ), but recently this value has been challenged. Since (at least for systems with small net baryon density such as those created in heavy-ion collisions at Relativistic Heavy Ion Collider (RHIC) energies near midrapidity) the phase transition is not a sharp singularity but rather a continuous cross-over where thermodynamic quantities change dramatically over a relatively narrow temperature range [2], the definition of $T_{c}$ is not unique, and one obtains slightly different values from different types of observables [3]. Depending on the choice of Lattice QCD action and extrapolation to the continuum, different groups have recently obtained $T_{c}$ values ranging from $T_{c}\left(\chi_{\bar{\psi} \psi}\right)=151 \pm 3 \pm 3 \mathrm{MeV}$ (extracted from the peak in the chiral susceptibility [3]) over $T_{c}\left(\chi_{s}\right) \approx T_{c}(P)=176 \pm 3 \pm 4 \mathrm{MeV}$ (extracted from the strange quark number and Polyakov loop susceptibilities [3]) to $T_{c}=192 \pm 7 \pm 4 \mathrm{MeV}$ (extracted from light quark number and Polyakov loop susceptibilities [4]). The differences between the numbers extracted by different groups [3,4] are larger than the quoted statistical and systematic errors and even exceed the FWHM widths $\Delta T_{c} \simeq 30-40 \mathrm{MeV}$ of the various susceptibilities [3]; they mostly reflect different choices for the observables used to set the physical scale and the procedures employed for extrapolating to zero lattice spacing $[3,4]$.

From experimental data collected in $\sqrt{s}=200 A \mathrm{GeV} \mathrm{Au}+\mathrm{Au}$ collisions at RHIC we know that the final hadron abundances from central collisions can be described by a hadron resonance gas in a state of approximate chemical equilibrium at $T_{\text {chem }}=163 \pm 4 \mathrm{MeV}, \mu_{B}=24 \pm 4 \mathrm{MeV}$, and a strangeness saturation factor $\gamma_{s}=0.99 \pm 0.07$ [5]. The quality of the statistical model fit is impressive. Furthermore, the STAR collaboration studied the dependence of the fit parameters on the collision centrality and found that neither the temperature $T_{\text {chem }}$ nor the baryon chemical potential $\mu_{B}$ depend appreciably on the impact parameter [6] ${ }^{1}$; only the strangeness suppression factor exhibits centrality dependence, beginning at impact parameters $>8-9 \mathrm{fm}$, and drops to values around 0.55 in the most peripheral $\mathrm{Au}+\mathrm{Au}$ collisions [5]. The centrality independence of $T_{\text {chem }}$ is in stark contrast to the behavior observed in the same experiment for the kinetic decoupling temperature $T_{\text {kin }}$ which is extracted (together with a value for the average radial flow velocity $\langle\beta\rangle$ of the fireball at kinetic freeze-out) from the shape of the transverse momentum spectra of identified pions, kaons and (anti-)protons [6]: $T_{\text {kin }}$ increases significantly with increasing impact parameter, from $T_{\text {kin }}=89 \pm 12 \mathrm{MeV}$ in the most central to $T_{\text {kin }}=127 \pm 13 \mathrm{MeV}$ in the most peripheral collisions. At the same time the average radial flow decreases from $\langle\beta\rangle=0.59 \pm 0.05$ in the most central to $\langle\beta\rangle=0.24 \pm 0.08$ in the most peripheral $\mathrm{Au}+\mathrm{Au}$ collisions, demonstrating a strong centrality dependence of the fireball expansion dynamics.

\footnotetext{
${ }^{1}$ The chemical decoupling temperatures extracted from the measured hadron abundance ratios depend somewhat on the details of the hadron resonance gas model employed: Ref. [5] gives $T_{\text {chem }}=163 \pm 4 \mathrm{MeV}, \mu_{B}=24 \pm 4 \mathrm{MeV}$ for central Au+Au collisions whereas in Ref. [6] one finds $T_{\text {chem }}=157 \pm 6 \mathrm{MeV}, \mu_{B}=22 \pm 4 \mathrm{MeV}$ for the same data. We will here use the values from the centrality dependence study presented in Ref. [6] which are consistently on the lower end of this range.
} 
This characteristic difference in the centrality dependences of the chemical and kinetic decoupling temperatures will be the main focus of this contribution. We will show that the observed centrality dependences of the average radial flow velocity and kinetic freeze-out temperature are consistent with hydrodynamic behaviour of the fireball medium followed by kinetic decoupling of the hadrons from microscopic scattering processes, driven by the collective expansion. We will then show that a centrality independent freeze-out temperature is inconsistent with a kinetic decoupling process unless the chemical scattering rates have an extremely (namely almost infinitely) strong temperature dependence. We interpret this finding as evidence that chemical decoupling of the hadron abundances is driven by a phase transition during which the chemical reaction rates decrease precipitously, leaving the system in a chemically frozen-out state at the end of the phase transition. Only in this way is it possible to obtain a universal chemical freeze-out temperature that is insensitive to the (centrality dependent) collective dynamics and only depends on the thermodynamic parameters of the phase transition. Obviously, the chemical processes happening during the hadronization process itself involve colored degrees of freedom and can thus not be efficiently described in hadronic language. We also address the centrality dependence of the strangeness saturation factor and comment on how our picture also reproduces chemical abundance data measured in $p p$ and $e^{+} e^{-}$collisions.

\section{Review of proposed phase transition signatures}

In this section we will review several previously published suggestions for QGP phase transition signatures in heavy-ion collisions and explain why they either failed or their status has remained ambiguous.

\subsection{Characteristics of the quark-hadron phase transition}

- Lattice QCD tells us [1] that the finite temperature, $\mu_{B} \approx 0$ QCD phase transition is associated with a rapid rise of the normalized energy and entropy densities, $e / T^{4}$ and $s / T^{3}$, near $T_{c}$, indicating a sudden change of the number of effective degrees of freedom.

- The square of the speed of sound $c_{s}^{2}=\frac{\partial p}{\partial e}$ exhibits a deep minimum near $T_{c}$, dropping from close to the ideal gas value $c_{s}^{2}=\frac{1}{3}$ at $T \gtrsim 2 T_{c}$ to almost $1 / 10$ of that value near $T_{c}$ [1] before rising again below $T_{c}$ to about half the ideal gas value, as calculated from the hadron resonance gas model [7]. (This is followed by a final exponential decrease as $T \rightarrow 0$ which is, however, not phenomenologically relevant since the fireball matter freezes out before reaching such low temperatures).

- Thermal fluctuations of the net electric charge and baryon number decrease by a factor 2-3 above $T_{c}$, as a result of the charge and baryon number being distributed in smaller (fractional) units.

- The transport properties of the matter, such as color conductivity $\sigma^{a b}$ [9] and specific shear viscosity $\eta / s[10]$, change dramatically at $T_{c}$. 
- Finally, chemical equilibration rates, in particular for processes involving the creation and destruction of strange quark pairs [11], speed up considerably above $T_{c}$, due to reduced mass thresholds as a result of chiral symmetry restoration.

All these changes refer to thermal equilibrium properties of the matter created in the collision, with the temperature as control parameter. Unfortunately, near $T_{c}$ a few percent rise of the temperature requires a severalfold increase of the energy density, so these changes set in much more slowly when viewed as a function of experimental conditions such as the collision energy, and it may be hard to recognize them among other effects. Finally, the medium produced in the collision cools through the phase transition quite rapidly, and even if it manages to remain near thermal equilibrium in spite of its strong collective dynamics the question remains which of these phase transition characteristics survive the expansion and affect the final hadronic state after freeze-out in a recognizable fashion.

\subsection{A visit to the graveyard of phase transition signatures}

The first attempt to connect the rapid rise of the entropy density $s$ at $T_{c}$ with experimental data was made by van Hove [12] who suggested to plot the average transverse momentum $\left\langle p_{T}\right\rangle$ of final state hadrons (as a proxy for the temperature) against their rapidity density $d N / d y$ (as a proxy for entropy density [13]) - the phase transition should then show up as a "plateau", i.e. as a (limited) region where $\left\langle p_{T}\right\rangle \sim T$ remains constant while the entropy density $s \sim\left(1 / \pi R_{A}^{2}\right)(d N / d y)$ keeps rising with increasing collision energy. However, McLerran et al. soon realized [14] that the average transverse momentum $\left\langle p_{T}\right\rangle$ of the final hadrons reflects a combination of random thermal and outwardly directed collective motion at freeze-out and is thus not a good proxy for the initial fireball temperature. Due to the collective flow component, $\left\langle p_{T}\right\rangle$ depends on the hadron rest mass, and since larger initial entropy or energy densities (generated by higher beam energies) increase the lifetime of the fireball before freeze-out, this collective flow component keeps increasing as the beam energy is raised even when the initial fireball temperature hardly changes inside the phase transition. As a result, there is no "plateau" of $\left\langle p_{T}\right\rangle$ vs. $d N / d y$ - the average transverse momentum increases monotonically with $d N / d y$, due to the monotonic increase of the collective radial flow with collision energy [14]. This growth is somewhat slowed in the phase transition region where the thermal $\left\langle p_{T}\right\rangle$ component remains constant, but that is difficult to observe, and it is even harder then to make a convincing case that such a change of slope in $\left\langle p_{T}\right\rangle$ vs. $d N / d y$ is indeed caused by a phase transition.

The speed of sound of the fireball medium represents its "pushing power", i.e. its ability to accelerate its collective flow in response to pressure gradients. Near a phase transition it becomes small (or even vanishes if the transition is of first order), thus it has been suggested early on (especially by W. Greiner, H. Stöcker and collaborators [15]) that phase transitions should be visible in the collective flow pattern of the matter created in heavy ion collisions. A specific suggestion made by Rischke et al. [16] within a 1-fluid hydrodynamic model was that the directed flow ("bounceoff"), when measured as a function of beam energy, should collapse around $E_{\text {lab }}=5 \mathrm{AGeV}$ due to the softness of matter created near the phase coexistence region, but recover again at higher beam energies when the fireball is initially in the QGP phase. Subsequent studies within 3-fluid hydrodynamics [17], where the two colliding nuclei and the matter created by their collision are 
described as coupled but separately thermalized fluids, showed that this phase transition signature is fragile and exhibits great sensitivity to the non-equilibrium dynamics describing the transfer of energy between the three fluids. In a realistic scenario the characteristic minimum in the excitation function of the directed flow completely disappears [17].

A variation of this theme was proposed in [18] whose authors suggested that the softness of the Equation of State (EoS) near a phase transition would manifest itself in a non-monotonic elliptic flow excitation function, with a minimum at collision energies high enough to initialize the medium slightly above the phase transition such that most of the elliptic flow would develop while the matter cools through the softest point. The advantage of this suggestion was that elliptic flow would be created only after the matter has thermalized, thereby avoiding the very early pre-equilibrium stage that proved fatal for the directed flow collapse. However, the elliptic flow minimum turned out to be fragile, too, only this time the phase transition signal was killed by non-equilibrium effects during the late instead of the early collision stages [19, 20]: At beam energies below the predicted minimum, the elliptic flow would have to be created in the hadron resonance gas phase (whose EoS is less stiff than that of the QGP but still much stiffer than the matter near the phase transition), but the latter is so viscous that its elliptic flow response to anisotropic pressure gradients is dramatically reduced. As a result, the elliptic flow signature never recovers at lower beam energies, thereby wiping out the predicted flow minimum around RHIC energies.

Thus, even though there is now strong evidence from a large variety of measurements that the dense matter created in Au+Au collisions at RHIC is initially in the QGP phase [21], no direct evidence exists that it indeed passes through a phase transition on its way to the final state of frozenout hadrons. Attempts to capitalize on the predicted change in the net charge and baryon number fluctuation spectrum [8] have not paid off - it seems that the predicted reduction in event-by-event fluctuations is largely washed out by the hadronization process.

Much attention has been paid to non-monotonicities in the excitation functions of several heavy-ion observables measured by the NA49 Collaboration in $\mathrm{Pb}+\mathrm{Pb}$ collisions at low SPS energies: a "kink" in the pion production per wounded nucleon, a "step" in the slope parameter of the $\mathrm{K}^{+}$transverse momentum spectrum, and a "peak" in the ratio of produced strange to non-strange quark-antiquark pairs (for a review see Ref. [22]). The suggested interpretation of these observations in terms of a phase transition $[23,22]$ is controversial since it relies on a number of unverivied simplifying assumptions and is so far not backed up by dynamical model calculations.

All the above suggestions are based on measuring excitation functions in order to steer the medium in a controlled way through the phase transition. The extraction of $T_{c}$ from such studies requires a model to quantitatively relate the control parameters $\sqrt{s}$ or $d N / d y$ to the initial energy density (and thus, via the assumption of thermalization), to a temperature).

We will here propose a different approach that exploits the centrality dependence of observables at fixed collision energies. While it has been pointed out repeatedly that the initial energy density of the created matter can be varied either by changing the collision energy for fixed collision system or by changing the size of the colliding nuclei or their impact parameter at fixed collision energy, we are not relying primarily on a controlled change of the initial energy density, but on the associated variation of the collective expansion rate. In order to explain how we use this, let us first discuss the recent controversy relating to the interpretation of chemical freeze-out data from relativistic heavy-ion collisions which motivated our work. 


\subsection{The controversy: Chemical reaction kinetics vs. statistical hadronization}

The hadrons emitted in relativistic heavy-ion collisions show thermal characteristics both in their abundances and in the shapes of their transverse momentum spectra, but the temperatures extracted from yields $[5,6,24,25]$ ("chemical freeze-out temperature" $T_{\text {chem }}$ ) and from spectra $[6,26,27,28,29]$ ("kinetic decoupling temperature" $T_{\text {kin }}$ ) differ significantly. The kinetic decoupling temperatures depend on beam energy and, at all beam energies, on system size and collision centrality, while for $\sqrt{s} \gtrsim 10 A \mathrm{GeV}$ approximately the same "universal" chemical freeze-out temperature is observed in $e^{+} e^{-}, p \bar{p}$ and $A+A$ collisions at all collision centralities (only the strangeness saturation factor $\gamma_{s}$ varies with collision system and centrality) [30].

This universal value $T_{\text {chem }}=160-170 \mathrm{MeV}$ is remarkably close to the critical temperature for the quark-hadron transition from Lattice QCD. Furthermore, kinetic simulations of the hadronic rescattering stage after QGP hadronization with hadronic cascade codes (RQMD, UrQMD, etc.) have shown that hadronic rescattering alters the momentum distributions and resonance populations through resonance scattering, thereby cooling the system while keeping it (at least for a while) close to local thermal equilibrium [31], while leaving the final stable hadron yields (after resonance decays) almost unaffected $[32,33]$. Hadronic rescattering leads to the loss of a fraction of the baryon-antibaryon pairs, but this can be at least partially traced back to the absence of multi-hadron collision channels so that detailed balance is violated in baryon-antibaryon annihilation channels $[34,35]$.

These empirical facts have split the heavy-ion theory community into two camps which offer different interpretations of the observations. The philosophy of Camp I is laid out in Refs. [30] (second paper) and $[36,33,37]$ and holds that hadron production is a statistical process associated with a phase transition, proceeding through very many different possible microscopic channels constrained only by energy, baryon number and strangeness conservation, thereby leading to a maximum entropy configuration described by a thermal distribution of hadron yields, with $T_{\text {chem }}$, $\mu_{B}$ and $\gamma_{s}$ playing the role of Lagrange multipliers to ensure these conservation law constraints while maximizing the entropy. The value of $T_{\text {chem }}$ is not established by inelastic reactions among hadrons proceeding until chemical equilibrium is reached - rather, the hadrons are directly "born" into a maximum entropy state of apparent chemical equilibrium [37], with the parameter $T_{\text {chem }}$ defining the critical energy density at which the hadronization process happens ([30]b). $T_{\text {chem }}$ is thus conceptually different from the kinetic decoupling temperature $T_{\text {kin }}$ which $i s$ the result of quasi-elastic rescattering among the hadrons (which also contribute to their collective flow).

Camp II includes the followers of Refs. [34, 35, 38, 39] who hold that chemical freeze-out is a kinetic process within the hadronic phase, conceptually equivalent with kinetic freeze-out, the only difference being the quantitative values of the corresponding freeze-out temperatures which reflect the fact that the inelastic cross sections driving chemical equilibration constitute only a small fraction of the total scattering cross section contributing to momentum exchange. The hadrons are not born into chemical equilibrium, but driven into such a state kinetically by inelastic multihadron processes (which, according to Refs. [34, 35, 38, 39], become crucial near $T_{c}$ due to high hadron densities) and frozen out by global expansion. Accordingly, $T_{\text {chem }}$ is the "real" temperature describing the latest point at which forward and backward chemical reactions balance each other. (In contrast, for Camp I, there are no "backward" reactions involving hadrons in both initial and 
final states.)

Is this more than a philosophical difference of opinions? We think so. Camp II has to cope with an intrinsic tension between two observations: The high quality of the thermal model fit to the observed hadron yields at RHIC requires sufficient time for inelastic reactions to establish chemical equilibrium, whereas the proximity of the fitted chemical freeze-out temperature $T_{\text {chem }}$ to the critical temperature $T_{c}$ of the quark-hadron phase transition from Lattice QCD, together with the rapid cooling of the fireball by collective expansion, don't provide much of a window for these processes to play out. In essence, to make the kinetic chemical equilibration scenario work one needs very large scattering rates right near $T_{c}$ which then drop to negligible values just below $T_{c}$. This would be easier to understand if there were a larger gap between $T_{c}$ and $T_{\text {chem }}$, as suggested by the recent upward revision of $T_{c}$ from Lattice QCD advocated in [4], but this problem appears serious if the lower $T_{c}\left(\chi_{\bar{\psi} \psi}\right)$ from Ref. [3] turns out to be correct.

In addition, we point out another conceptual problem with the interpretation by Camp II: If freeze-out is a kinetic process, it is controlled by the competition between local scattering (moving the system towards equilibrium) and global expansion (driving the system out of equilibrium). The resulting freeze-out temperature is therefore sensitive to the fireball expansion rate. We show that the latter depends on collision centrality. Therefore, any kinetic freeze-out temperature must depend on impact parameter. While this is empirically indeed the case for the kinetic decoupling temperature $T_{\text {kin }}$, the chemical freeze-out temperature does not seem to vary with collision centrality. Hence it cannot be the result of a kinetic decoupling process from inelastic hadronic scattering.

\section{Kinetic freeze-out from a hydrodynamically expanding system}

In this section we show that the hydrodynamic model can quantitatively reproduce the observed centrality dependence of the kinetic decoupling temperature extracted from hadron momentum spectra at RHIC. We then show that an analogous centrality dependence of the chemical freeze-out temperature cannot be avoided if the hadron yields are similarly controlled by kinetic freeze-out from inelastic hadronic rescattering.

We use our (2+1)-dimensional longitudinally boost-invariant hydrodynamic code AZHYDRO [40] with standard initial conditions [7] to generate the flow pattern for $200 \mathrm{~A} \mathrm{GeV} \mathrm{Au+Au} \mathrm{colli-}$ sions. This code has been previously shown to successfully reproduce the measured single particle hadron $p_{T}$-spectra and their elliptic flow (for details see [41]). Here, however, we modify the freeze-out criterium for kinetic decoupling to account for its kinetic nature: Instead of requiring freeze-out on a surface of constant energy density $e_{\mathrm{dec}}=0.075 \mathrm{GeV} / \mathrm{fm}^{3}$ (corresponding to a fixed temperature $T_{\text {kin }}=100 \mathrm{MeV}$ [7]), we define the kinetic freeze-out surface as the set of points where the local expansion rate $1 / \tau_{\exp }(x)=\partial \cdot u(x)$ (where $u^{\mu}(x)$ is the hydrodynamic flow 4-velocity) becomes equal to the local scattering rate $1 / \tau_{\text {scatt }}(x)[42,43,44]$ :

$$
\frac{1}{\tau_{\text {scatt }}}=\xi \frac{1}{\tau_{\exp }}=\xi \partial \cdot u(x) \text {. }
$$

Here $\xi$ is a proportionality constant of order unity which we set in a first attempt to $\xi=0.35$, yielding an average temperature along the freeze-out surface for central $\mathrm{Au}+\mathrm{Au}$ collisions of $\left\langle T_{\mathrm{kin}}\right\rangle \simeq$ $115 \mathrm{MeV}$. Having fixed $\xi$ in central collisions, Eq. (3.1) is taken to define the freeze-out surface 
also at other impact parameters. Inside the freeze-out surface the scattering rate exceeds $\xi$ times the expansion rate, and the matter is thermalized, whereas outside the surface the expansion rate exceeds $\xi^{-1}$ times the scattering rate so we assume the hadrons there to be decoupled from the fluid, streaming freely into the detector.

The expansion rate $\partial \cdot u=\gamma_{\perp}\left(\frac{1}{\tau}+\nabla_{\perp} \cdot v_{\perp}\right)+\left(\partial_{\tau}+\boldsymbol{v}_{\perp} \cdot \nabla_{\perp}\right) \gamma_{\perp}$ is computed from the hydrodynamic output for the transverse flow velocity $\boldsymbol{v}_{\perp}(x)$ and $\gamma_{\perp}=\left(1+v_{\perp}^{2}\right)^{-1 / 2}$.

The scattering rate in Eq. (3.1) involves cross sections and densities of scatterers, hence it is particle specific $[43,44]$. Since at RHIC energies hadron production is dominated by pions, we assume for simplicity that all hadrons decouple when pions freeze out. It may be somewhat better to describe different hadron species by different freeze-out temperatures [44], but each of them would exhibit similar impact parameter dependences as the one for pions which we work out here.

The pion scattering rate we take from the numerical results presented in Ref. [44] which we parametrize as

$$
\frac{1}{\tau_{\text {scatt }}^{\pi}}=\left(59.5 \mathrm{fm}^{-1}\right)\left(\frac{T}{1 \mathrm{GeV}}\right)^{3.45} \text {. }
$$

This defines the momentum exchange rate to be used for the calculation of kinetic freeze-out and will need to be modified when discussing chemical freeze-out below.

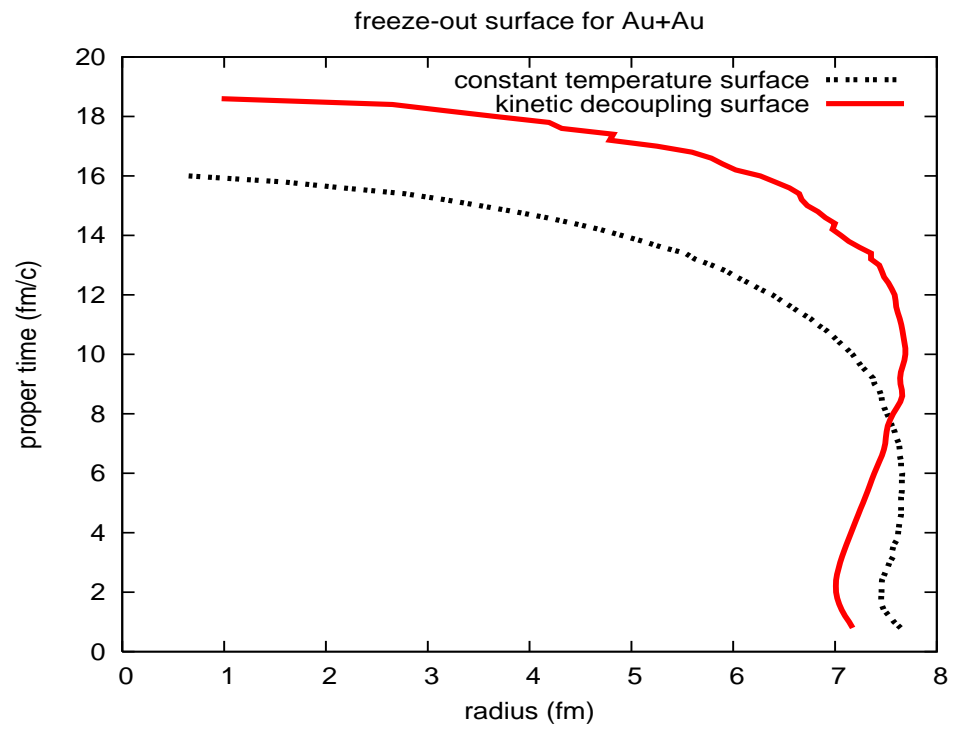

Figure 1: Kinetic freeze-out surface $\tau_{\text {kin }}(r)$ for central $(b=0) 200 \mathrm{~A} \mathrm{GeV} \mathrm{Au+Au} \mathrm{collisions,} \mathrm{computed} \mathrm{from}$ Eq. (3.1) with $\xi=0.35$ (red solid line) and for a constant freeze-out temperature $T_{\text {kin }}=115 \mathrm{MeV}$ (dotted black line). Both surfaces have the same average temperature of $\langle T\rangle=115 \mathrm{MeV}$, using the energy density as weight function.

In Fig. 1 we show the kinetic freeze-out surface for central Au+Au collisions computed from Eq. (3.1) with $\xi=0.35$ (solid red line) and from the condition $T_{\text {kin }}=115 \mathrm{MeV}$ (dotted black line). Both have the same average kinetic freeze-out temperature, but for the kinetic freeze-out criterium (3.1) the middle of the fireball freezes out a bit later at lower temperature and larger flow whereas the edge decouples earlier at higher temperature and with less flow than the contant- $T$ surface. This is caused by the larger expansion rate near the edge of the fireball. 

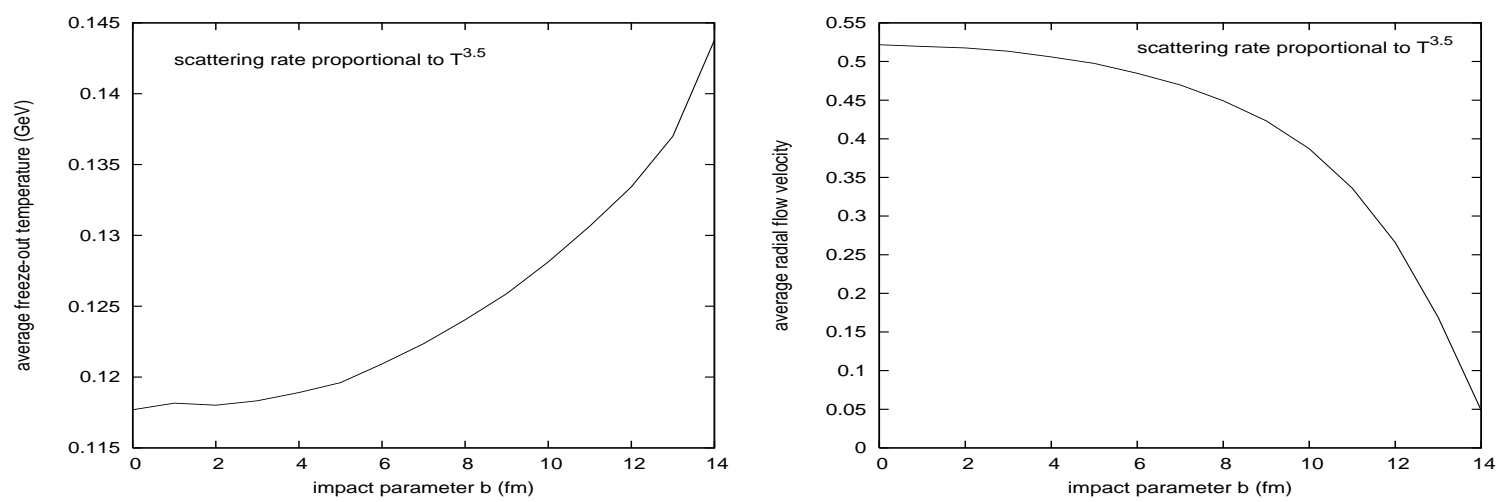

Figure 2: Impact parameter dependence of the average kinetic decoupling temperature $\left\langle T_{\mathrm{kin}}\right\rangle$ (left) and average radial flow velocity $\left\langle v_{\perp}\right\rangle$ along the freeze-out surface computed from Eq. (3.1) with $\xi=0.35$ and a scattering rate $\sim T^{3.45}$ as in Eq. (3.2), for $200 \mathrm{~A} \mathrm{GeV} \mathrm{Au+Au} \mathrm{collisions.}$

Figure 2 shows the impact parameter dependence of the average kinetic decoupling temperature and the associated average radial flow calculated from the kinetic freeze-out criterium (3.1) with $\xi=0.35$. One sees that central collisions decouple at relatively low temperatures with large average radial flow whereas peripheral collisions freeze out earlier when the fireballs are still hotter and less radial flow has developed. This is in good qualitative agreement with the STAR data in Ref. [6], although there the freeze-out temperatures are generally a bit lower, with slightly larger average radial flow velocities than seen in Fig. 2. We adjust for this by fine-tuning the phenomeno-

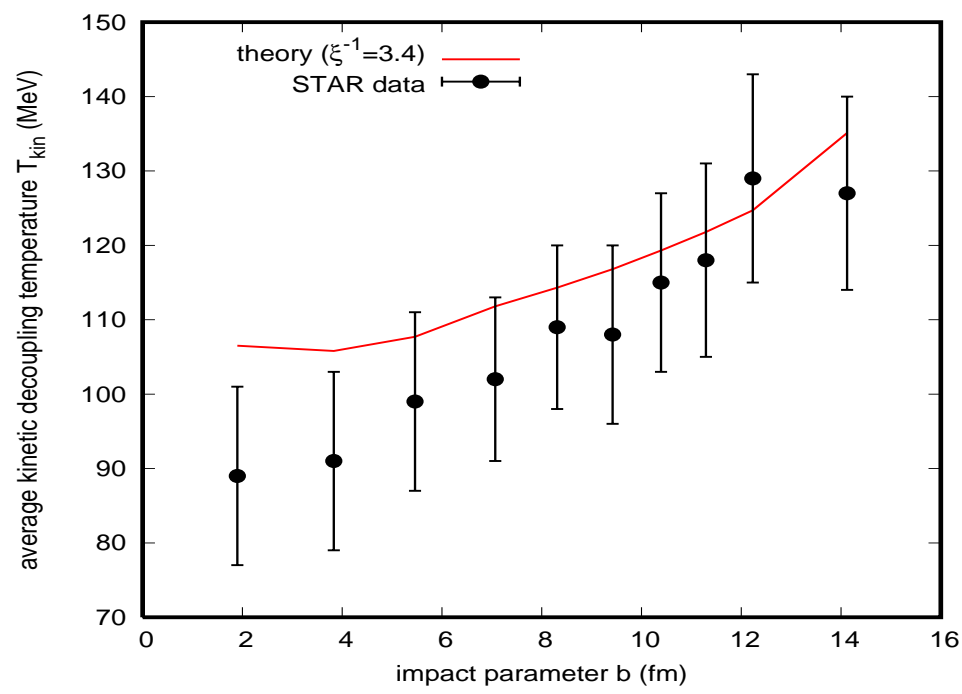

Figure 3: Impact parameter dependence of the average kinetic decoupling temperature $\left\langle T_{\text {kin }}\right\rangle$ computed from hydrodynamics with kinetic freeze-out criterium (3.1) using $\xi=0.295$, compared with STAR data [6] for $200 \mathrm{~A} \mathrm{GeV} \mathrm{Au+Au} \mathrm{collisions.}$

logical parameter $\xi$ in Eq. (3.1) to $\xi=0.295\left(\xi^{-1}=3.4\right)$. The corresponding freeze-out temperatures are shown as a function of impact parameter $b$ in Figure 3, together with the STAR data. Now the agreement is also quantitatively acceptable. We conclude that the measured centrality dependence of $T_{\text {kin }}$ can be completely understood in terms of a hydrodynamic model for the fireball 
expansion coupled to a kinetic freeze-out criterium with realistic temperature dependence of the microscopic scattering rate.

Let us now see whether we can similarly understand chemical freeze-out as a kinetic decoupling process from inelastic hadronic scattering. A few typical processes relevant for chemical equilibration are

$$
\begin{array}{lcl}
\pi+\pi \longleftrightarrow K+\bar{K}, & \pi+N \longleftrightarrow K+Y,, & \pi+Y \longleftrightarrow \bar{K}+N, \\
\Omega+\pi \longleftrightarrow \Xi+\bar{K}, & K+\bar{K} \longleftrightarrow \phi+\pi, & \Omega+\bar{K} \longleftrightarrow \Xi+\pi, \\
\Omega+\bar{N} \longleftrightarrow 2 \pi+3 \bar{K}, & N+\bar{N} \longleftrightarrow 5 \pi, & N+3 \bar{K} \longleftrightarrow \Omega+3 \pi .
\end{array}
$$

The last line shows so-called multi-hadron collision channels which in at least one direction require collisions between more than two hadrons. Rates for processes involving $n_{\text {in }}$ incoming hadrons are proportional to the product of their densities $\sim \prod_{i=1}^{n_{\text {in }}} n_{i}(T)$ where each factor $n_{i}(T)$ grows with $T$ at least as $T^{3}$ (even much more rapidly for hadrons with masses $>T$ ). At low temperatures, multi-hadron collision processes as well as collisions between very massive hadrons are therefore strongly suppressed. Consequently, particle yields for hadrons requiring collisions of many abundantly available particles for their production or destruction (such as $\bar{p}, \Omega, \ldots$ ) thus tend to freeze out at higher $T$ than particle yields for hadrons whose abundances can be efficiently changed by two-body reactions $(\pi, K, \phi, \ldots)$.

In an expanding, cooling system, simultaneous freeze-out of all hadron yields at a common temperature therefore requires a conspiracy of rates with widely differring $T$-dependences. Indeed, thermal model fits to hadron abundances with a single common temperature are usually not perfect [45], and individual fits to subsets of yields measured in lower-energy collisions at the SPS and AGS tend to lead to a significant spread of chemical freeze-out temperatures [46]. Only at RHIC energies so far the single-temperature chemical equilibrium fit gives an almost perfect fit to the data $[25,46]$.

One way to achieve the conspiracy of different chemical equilibration rates that is required for a good fit with a single freeze-out temperature is to postulate that at chemical freeze-out all chemical reactions are completely dominated by multi-hadron collisions and that at any temperature below $T_{\text {chem }}$ the medium is so rarefied and so rapidly expanding that even the simplest two-body reactions among the most abundantly produced hadrons (such as those listed in the first line of Eq. (3.3)) have essentially stopped. As long as collision channels with widely different temperature dependences compete with each other, chemical freeze-out of all hadron species at a single temperature appears to impossible.

Even more importantly, even if it were possible at one fixed impact parameter to arrange for common freeze-out of all hadron species in spite of a competition of scattering rates with different temperature dependences, such a conspiracy would be impossible to maintain, with the same value for the freeze-out temperature $T_{\text {chem }}$, over the entire impact parameter range. Figure 4 shows the centrality dependence of the average chemical freeze-out temperatures along hydrodynamic decoupling surfaces computed with the kinetic freeze-out criterium (3.1), using $\xi=0.95$ to adjust the value of $\left\langle T_{\text {chem }}\right\rangle$ in central Au+Au collisions to the STAR data [6] and exploring different possible temperature dependences of the dominant inelastic scattering rate. One sees that approximate im- 


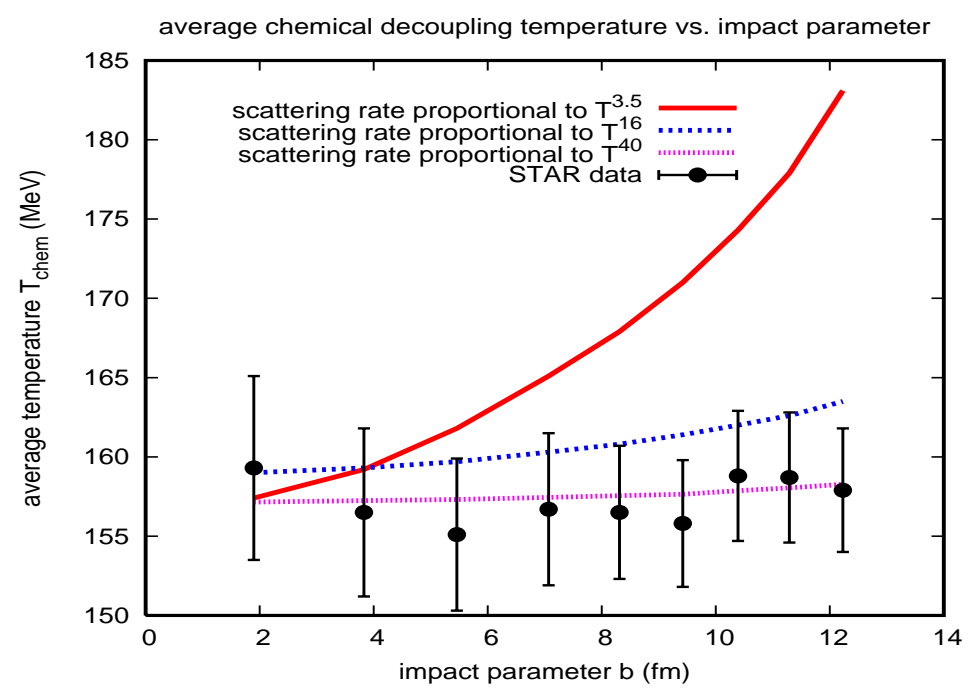

Figure 4: Impact parameter dependence of the average chemical decoupling temperature $\left\langle T_{\text {chem }}\right\rangle$ computed from hydrodynamics with kinetic freeze-out criterium (3.1) using $\xi=0.95$ and reaction rates with different temperature dependences as listed, compared with STAR data [6] for $200 \mathrm{~A} \mathrm{GeV} \mathrm{Au+Au} \mathrm{collisions.}$

pact parameter independence of $\left\langle T_{\text {chem }}\right\rangle$ can only be achieved if all inelastic scattering rates grow with $T$ as $T^{n}$ with a power $n \gtrsim 20$ !

Basically Figure 4 tells us that the observed centrality independence of $T_{\text {chem }}$ requires chemical freeze-out to happen in a region of parameter space where all chemical reaction rates exhibit extremely steep temperature dependence, dropping like a stone as the system cools through the decoupling temperature. It is hard to understand such a behavior within a hadron rescattering picture unless one assumes that all relevant chemical reactions involve multi-particle channels involving many hadrons. Making such an assumption clearly pushes the hadronic rescattering model towards breakdown because its chemical kinetics would essentially be controlled by interactions among clusters of particles involving an unspecifiable number of hadrons. It is much more natural to associate this kind of behavior with the quark-hadron phase transition where densely spaced and strongly interacting quarks and gluons provide the necessary multi-particle clusters, and where the dramatic change in number and quality of the effective degrees of freedom within a narrow temperature interval generates the dramatic temperature dependence of the chemical reaction rates at decoupling which seem to be phenomenologically required.

In such a picture, hadrons are not really well-defined states until after the quark-hadron phase transition is complete and, at the same time, chemical reactions among hadrons have ceased. Hadrons are thus indeed "born into chemical equilibrium" [37] in a process that can be rightfully called "statistical hadronization" [30, 36, 33]. If hadrons are formed in this fashion, their measured abundances provide a window with a direct view of the QCD quark-hadron phase transition.

\section{Conclusions}

We have shown that the observed impact parameter dependence of the average temperature and radial flow velocity at kinetic (thermal) freeze-out (i.e. at the point where the hadron mo- 
mentum distributions decouple) can be quantitatively understood as a kinetic decoupling process in a hydrodynamically expanding source, with freeze-out being driven by the global expansion of the collision fireball. Any such kinetic decoupling process is controlled by the local competition between temperature dependent scattering and hydrodynamic expansion rates, and since the latter change with impact parameter as a result of the varying initial energy density and size of the nuclear collision zone, the resulting average freeze-out temperature is necessarily impact parameter dependent. The strength of this impact parameter dependence (i.e. the sensitivity of the freeze-out temperature to the fireball expansion rate) is inversely related to the strength of the temperature dependence of the local scattering rate. To obtain approximate centrality independence of the freeze-out temperature, the scattering rate must exhibit an almost infinitely steep temperature dependence.

From this it follows that the observed impact parameter independence of the chemical freezeout temperature in $\mathrm{Au}+\mathrm{Au}$ collisions at RHIC (i.e. of the temperature where the abundances of stable hadron species decouple) cannot be consistently described as the result of a kinetic decoupling process from inelastic hadronic interactions. To obtain the necessary extremely steep temperature dependence of the inelastic scattering rate $\left(\sim T^{n}\right.$ with $\left.n \gtrsim 20\right)$ requires that at the freeze-out point all chemical reactions are dominated by multi-hadron interactions involving many more than two colliding particles, in which case it seems unlikely that one will ever be able to describe this process quantitatively in hadronic language.

In our opinion the only theoretically consistent interpretation of the STAR data on chemical freeze-out is to associate the steepness of the the temperature dependence of chemical equilibration rates with a phase transition (in this case the quark-hadron transition) in which the hadrons are produced statistically and distributed among different species according to the principle of maximum entropy, via a multitude of complicated microscopic channels involving large numbers of strongly interacting quarks and gluons. In this sense the hadrons are "born into chemical equilibrium" in an environment that is too dilute and expands too rapidly to allow for any further inelastic reactions among the hadrons.

$T_{\text {kin }}$ and $T_{\text {chem }}$ thus stand on conceptually different footings. $T_{\text {chem }}$ is a Lagrange multiplier related by the Maximum Entropy Principle to the critical energy density $e_{c}$ for hadronization. Its universality in $e^{+} e^{-}, p p$, and $A A$ collisions of all centralities shows that at $e_{c}$ a phase transition occurs. Hadrons are formed during this transition in a statistical process subject to the Principle of Maximum Entropy.

The absence of inelastic hadronic rescattering processes allows the direct measurement of $T_{c}$ through $T_{\text {chem }}$ and thus the experimental observation of the phase transition. In this context the question arises which of the different definitions of the critical temperature $T_{c}$ from Lattice QCD that were mentioned in the Introduction is most closely related to the chemical freeze-out temperature $T_{\text {chem }}$ extracted from hadron yield data. It seems unlikely that hadron yields can be considered frozen out before the hadrons have more or less recovered their full vacuum masses, and this is related to the restoration of the chiral condensate $\langle\bar{\psi} \psi\rangle$ to its vacuum value. We therefore suggest that $T_{c}\left(\chi_{\bar{\psi} \psi}\right)$ [3] should be the LQCD number most closely related to the phenomenological value $T_{\text {chem. }}$. This seems to be consistent with the actual values extracted in [3] from LQCD and in [6] from hadron yields at RHIC (both are between 150 and $160 \mathrm{MeV}$ ).

The increase of the strangeness saturation factor $\gamma_{s}$ from $e^{+} e^{-}$and $p p$ to heavy-ion colli- 
sions and from peripheral to central $\mathrm{Au}+\mathrm{Au}$ collisions at RHIC shows that the lifetime of the QGP (and thus the time for chemically equilibrating strange with light quarks) is still limited. Only for midcentral to central $\mathrm{Au}+\mathrm{Au}$ collisions $\gamma_{s}$ has sufficient time to saturate. (Qualitatively similar tendencies are seen in $\mathrm{Pb}+\mathrm{Pb}$ collisions at lower SPS energies [25].) The primary parton production process at the beginning of the collision apparently suppresses the production of strange quarks, and it also produces $s$ and $\bar{s}$ locally in pairs, thereby generating spatial correlations among $s$ and $\bar{s}$ which ensure strangeness conservation locally. In a grand canonical description such correlations induce a strangeness suppression factor $\gamma_{s}<1$ [30]. It takes time to diffuse the strange quarks over the entire fireball volume to decorrelate them and adjust their abundance to equilibrium values. Larger initial energy densities in central $\mathrm{Au}+\mathrm{Au}$ collision provide more time until the point of hadronization at $e_{c} \simeq 0.7 \mathrm{GeV} / \mathrm{fm}^{3}$ is reached than peripheral $\mathrm{Au}+\mathrm{Au}$ or $e^{+} e^{-}$and $p p$ collisions.

We close by pointing out that our conclusions about the nature and origin of $T_{\text {chem }}$ can be put to a relatively easy experimental test: It is well known $[24,25]$ that at low SPS and AGS energies, where the net baryon density of the matter created in the collision is much larger than at RHIC, the measured chemical decoupling temperatures are well below generally accepted estimates for the phase transition temperature, $T_{\text {chem }}<T_{c}$. In that case the phase transition can not be the origin of the observation of chemical equilibrium yields; hadronic chemical reactions must be responsible for lowering the chemical freeze-out temperature to values significantly below $T_{c}$. Since the present work has shown that the kinetic decoupling of hadronic chemical reaction rates is influenced by the fireball expansion rate, which again depends on collision centrality, we expect to see impact parameter dependence of $T_{\text {chem }}$ whenever its value is measured to be well below $T_{c}$. This conclusion would also apply to RHIC collisions if Lattice QCD would eventually converge to $T_{c}$ values above $190 \mathrm{MeV}$ as proposed in [4]. In this case we would definitely expect $T_{\text {chem }}$ to depend on collision centrality and ask for a reanalyzis of chemical decoupling data at RHIC with higher statistics in order to unambiguously settle this question.

It may be possible to re-analyze existing SPS data to confirm or falsify our prediction of centrality dependence of $T_{\text {chem }}$ at these energies. If not, this will be a worthwhile point to address within the planned low-energy collision program at RHIC. Clarification of this point will be of utmost importance for establishing the observed chemical decoupling temperature at RHIC as a direct measurement of the critical temperature of the quark-hadron phase transition in QCD.

\section{References}

[1] F. Karsch and E. Laermann, Thermodynamics and in-medium hadron properties from lattice QCD, in Quark Gluon Plasma 3, edited by R. C. Hwa and X. N. Wang (World Scientific, Singapore, 2004), p. 1 [hep-lat/0305025].

[2] Y. Aoki, G. Endrodi, Z. Fodor, S.D. Katz and K.K. Szabo, The order of the quantum chromodynamics transition predicted by the standard model of particle physics, Nature 443 (2006) 675.

[3] Y. Aoki, Z. Fodor, S. D. Katz and K. K. Szabo, The QCD transition temperature: Results with physical masses in the continuum limit, Phys. Lett. B 643 (2006) 46.

[4] M. Cheng et al., The transition temperature in QCD, Phys. Rev. D 74 (2006) 054507. 
[5] J. Adams et al. [STAR Collaboration], Experimental and theoretical challenges in the search for the quark gluon plasma: The STAR Collaboration's critical assessment of the evidence from RHIC collisions, Nucl. Phys. A 757 (2005) 102.

[6] J. Adams et al. [STAR Collaboration], Identified particle distributions in pp and Au+Au collisions at $\sqrt{s_{N N}}=200 \mathrm{GeV}$, Phys. Rev. Lett. 92 (2004) 112301.

[7] P. F. Kolb and U. Heinz, Hydrodynamic description of ultrarelativistic heavy ion collisions, in Quark Gluon Plasma 3, edited by R. C. Hwa and X. N. Wang (World Scientific, Singapore, 2004), p. 634, and references therein.

[8] M. Asakawa, U. Heinz and B. Müller, Fluctuation probes of quark deconfinement, Phys. Rev. Lett. 85 (2000) 2072; S. Jeon and V. Koch, Charged particle ratio fluctuation as a signal for QGP, Phys. Rev. Lett. 85 (2000) 2076.

[9] U. Heinz, Quark-gluon transport theory. Part 2: Color response and color correlations in a quark-gluon plasma, Ann. Phys. (N.Y.) 168 (1986) 148.

[10] T. Hirano and M. Gyulassy, Perfect fluidity of the quark gluon plasma core as seen through its dissipative hadronic corona, Nucl. Phys. A 769 (2006) 71; L. P. Csernai, J. I. Kapusta and L. D. McLerran, On the strongly-interacting low-viscosity matter created in relativistic nuclear collisions, Phys. Rev. Lett. 97 (2006) 152303.

[11] J. Rafelski and B. Müller, Strangeness production in the quark-gluon plasma, Phys. Rev. Lett. 48 (1982) 1066 [Erratum ibid. 56 (1986) 2334].

[12] L. Van Hove, Multiplicity dependence of $p_{T}$ spectrum as a possible signal for a phase transition in hadronic collisions, Phys. Lett. B 118 (1982) 138.

[13] R. C. Hwa and K. Kajantie, Diagnosing quark matter by measuring the total entropy and the photon or dilepton emission rates, Phys. Rev. D 32 (1985) 1109.

[14] M. Kataja, L. McLerran, V. Ruuskanen and H. van Gersdorff, Studies of the hydrodynamical evolution of matter produced in fluctuations in $p \bar{p}$ and in ultrarelativistic nuclear collisions. 2. Transverse momentum distributions, Phys. Rev. D 34 (1986) 2755.

[15] J. Hofmann, H. Stoecker, U. Heinz, W. Scheid and W. Greiner, Possibility of detecting density isomers in high density nuclear Mach shock waves, Phys. Rev. Lett. 36 (1976) 88; H. Stoecker and W. Greiner, High-energy heavy ion collisions: probing the equation of state of highly excited hadronic matter, Phys. Rept. 137 (1986) 277.

[16] D. H. Rischke, Y. Pürsün, J. A. Maruhn, H. Stoecker and W. Greiner, The phase transition to the quark-gluon plasma and its effects on hydrodynamic flow, Heavy Ion Phys. 1 (1995) 309.

[17] J. Brachmann et al., Antiflow of nucleons at the softest point of the EoS, Phys. Rev. C 61 (2000) 024909.

[18] P. F. Kolb, J. Sollfrank and U. Heinz, Anisotropic transverse flow and the quark-hadron phase transition, Phys. Rev. C 62 (2000) 054909.

[19] D. Teaney, J. Lauret and E. V. Shuryak, A hydrodynamic description of heavy ion collisions at the SPS and RHIC, nucl-th/0110037.

[20] T. Hirano, U. Heinz, D. Kharzeev, R. Lacey and Y. Nara, Hadronic dissipative effects on elliptic flow in ultrarelativistic heavy-ion collisions, Phys. Lett. B 636 (2006) 299.

[21] U. Heinz, The quark-gluon plasma at RHIC, Nucl. Phys. A 721 (2003) 30. 
[22] P. Seyboth et al. [NA49 Collaboration], Indications for the onset of deconfinement in $\mathrm{Pb}+\mathrm{Pb}$ collisions at the CERN SPS from NA49, Acta Phys. Polon. B 36 (2005) 565.

[23] M. Gazdzicki, Observation of deconfinement phase transition in nucleus-nucleus collisions, Acta Phys. Polon. B 35 (2004) 187.

[24] J. Cleymans and K. Redlich, Unified description of freeze-out parameters in relativistic heavy ion collisions, Phys. Rev. Lett. 81 (1998) 5284.

[25] P. Braun-Munzinger, K. Redlich and J. Stachel, Particle production in heavy ion collisions, in Quark Gluon Plasma 3, edited by R. C. Hwa and X. N. Wang (World Scientific, Singapore, 2004), p. 491, and references therein.

[26] H. Dobler, J. Sollfrank and U. Heinz, Kinetic freeze-out and radial flow in $11.6 \mathrm{~A} \mathrm{GeV} \mathrm{Au+Au}$ collisions, Phys. Lett. B 457 (1999) 353.

[27] J. Cleymans and K. Redlich, Chemical and thermal freeze-out parameters from $1 \mathrm{~A} \mathrm{GeV} \mathrm{to} 200 \mathrm{~A} \mathrm{GeV \text {, }}$ Phys. Rev. C 60 (1999) 054908.

[28] B. Tomasik, U. A. Wiedemann and U. Heinz, Reconstructing the freeze-out state in $P b+P b$ collisions at 158 A GeV/c, Heavy Ion Phys. 17 (2003) 105.

[29] J.M. Burward-Hoy et al. (PHENIX Collaboration), Source parameters from identified hadron spectra and HBT radii for Au+Au collisions at $\sqrt{s_{N N}}=200 \mathrm{GeV}$ in PHENIX, Nucl. Phys. A 715 (2003) 498c.

[30] F. Becattini, A Thermodynamical approach to hadron production in $e^{+} e^{-}$collisions, Z. Phys. C 69

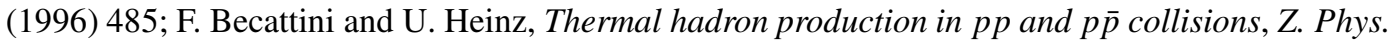
C 76 (1997) 269 [Erratum ibid. C 76 (1997) 578]; F. Becattini, M. Gazdzicki and J. Sollfrank, On chemical equilibrium in nuclear collisions, Eur. Phys. J. C 5 (1998) 143; F. Becattini, M. Gazdzicki, A. Keranen, J. Manninen and R. Stock, Study of chemical equilibrium in nucleus nucleus collisions at AGS and SPS energies, Phys. Rev. C 69 (2004) 024905.

[31] L. V. Bravina et al., Local equilibrium in heavy-ion collisions: Microscopic analysis of a central cell versus infinite matter, Phys. Rev. C 62 (2000) 064906; L. V. Bravina et al., Equation of state of resonance-rich matter in the central cell in heavy-ion collisions at $\sqrt{s}=200 \mathrm{~A} \mathrm{GeV}$, Phys. Rev. C 63 (2001) 064902.

[32] S. A. Bass, A. Dumitru, M. Bleicher, L. Bravina, E. Zabrodin, H. Stoecker and W. Greiner, Hadronic freeze-out following a first order hadronization phase transition in ultrarelativistic heavy-ion collisions, Phys. Rev. C 60 (1999) 021902; S. A. Bass and A. Dumitru, Dynamics of hot bulk QCD matter: From the quark-gluon plasma to hadronic freeze-out, Phys. Rev. C 61 (2000) 064909.

[33] U. Heinz, Primordial hadrosynthesis in the Little Bang, Nucl. Phys. A 661 (1999) 140.

[34] R. Rapp and E. V. Shuryak, Resolving the antibaryon production puzzle in high-energy heavy-ion collisions, Phys. Rev. Lett. 86 (2001) 2980.

[35] C. Greiner and S. Leupold, Antihyperon production in relativistic heavy ion collisions, J. Phys. G 27 (2001) L95.

[36] U. Heinz, Hadronic observables: Theoretical highlights, Nucl. Phys. A 638 (1998) 357c.

[37] R. Stock, The parton to hadron phase transition observed in Pb+Pb collisions at $158 \mathrm{GeV}$ per nucleon, Phys. Lett. B 456 (1999) 277.

[38] P. Braun-Munzinger, J. Stachel and C. Wetterich, Chemical freeze-out and the QCD phase transition temperature, Phys. Lett. B 596 (2004) 61. 
[39] C. Greiner, P. Koch-Steinheimer, F. M. Liu, I. Shovkovy and H. Stoecker, Chemical equilibration due to heavy Hagedorn states, J. Phys. G 31 (2005) S725.

[40] The code can be downloaded from URL http://nt3.phys.columbia.edu/people/molnard/OSCAR/. See also Ref. [18] and P. F. Kolb and R. Rapp, Transverse flow and hadrochemistry in Au+Au collisions at $\sqrt{s_{N N}}=200 \mathrm{GeV}$, Phys. Rev. C 67 (2003) 044903.

[41] U. Heinz, Hydrodynamics at RHIC: How well does it work, where and how does it break down?, J. Phys. G 31 (2005) S717; U. Heinz, 'RHIC serves the perfect fluid' - Hydrodynamic flow of the QGP, nucl-th/0512051.

[42] J. P. Bondorf, S. I. A. Garpman and J. Zimanyi, A simple analytic hydrodynamic model for expanding fireballs, Nucl. Phys. A 296 (1978) 320.

[43] E. Schnedermann, J. Sollfrank and U. Heinz, Fireball spectra, NATO Adv. Study Inst. Ser. B Phys. 303 (1993) 175; E. Schnedermann and U. Heinz, A hydrodynamical assessment of 200 A GeV collisions, Phys. Rev. C 50 (1994) 1675.

[44] C. M. Hung and E. V. Shuryak, Equation of state, radial flow and freeze-out in high energy heavy ion collisions, Phys. Rev. C 57 (1998) 1891.

[45] J. Sollfrank, U. Heinz, H. Sorge and N. Xu, Thermal analysis of hadron multiplicities from RQMD, Phys. Rev. C 59 (1999) 1637.

[46] A. Dumitru, L. Portugal and D. Zschiesche, Inhomogeneous freeze-out in relativistic heavy-ion collisions, Phys. Rev. C 73 (2006) 024902. 